\title{
An optimized high-performance liquid chromatography (HPLC) method for benzoylmesaconine determination in Radix Aconiti Lateralis Preparata (Fuzi, aconite roots) and its products Ying Xie ${ }^{1}$, Hua Zhou ${ }^{1}$, Yuen Fan Wong ${ }^{1}$, Zhongqiu Liu ${ }^{1}$, Hongxi Xu${ }^{2}$, Zhihong Jiang*1 and Liang Liu*1
}

Address: ${ }^{1}$ School of Chinese Medicine, Hong Kong Baptist University, Hong Kong SAR, PR China and ${ }^{2}$ Hong Kong Jockey Club Institute of Chinese Medicine, Hong Kong SAR, PR China

Email: Ying Xie - 04415930@hkbu.edu.hk; Hua Zhou - hz2009@hkbu.edu.hk; Yuen Fan Wong - ivanwong@hkbu.edu.hk; Zhongqiu Liu - liuzq@hkbu.edu.hk; Hongxi Xu - xuhongxi@hkjcicm.org; Zhihong Jiang* - zhjiang@hkbu.edu.hk; Liang Liu* - liuliang@hkbu.edu.hk

* Corresponding authors

Published: 30 May 2008

Chinese Medicine 2008, 3:6 doi:10.1 I86/1749-8546-3-6

This article is available from: http://www.cmjournal.org/content/3/I/6

(C) 2008 Xie et al; licensee BioMed Central Ltd.

This is an Open Access article distributed under the terms of the Creative Commons Attribution License (http://creativecommons.org/licenses/by/2.0), which permits unrestricted use, distribution, and reproduction in any medium, provided the original work is properly cited.
Received: 8 August 2006

Accepted: 30 May 2008

\begin{abstract}
Background: Benzoylmesaconine (BMA) is the main Aconitum alkaloid in Radix Aconiti Lateralis Preparata (Fuzi, aconite roots) with potent pharmacological activities, such as analgesia and antiinflammation. The present study developed a simple and reliable method using BMA as a marker compound for the quality control of processed aconite roots and their products.
\end{abstract}

Methods: After extraction, a high-performance liquid chromatography (HPLC) determination of BMA was conducted on a RP- $\mathrm{C}_{18}$ column by gradient elution with acetonitrile and aqueous phase, containing $0.1 \%$ phosphoric acid adjusted with triethylamine to $\mathrm{pH} 3.0$.

Results: A distinct peak profile was obtained and separation of BMA was achieved. Method validation showed that the relative standard deviations (RSDs) of the precision of BMA in all intraday and inter-day assays were less than $1.36 \%$, and that the average recovery rate was $96.95 \%$. Quantitative analysis of BMA showed that the content of BMA varied significantly in processed aconite roots and their products.

Conclusion: This HPLC method using BMA as a marker compound is applicable to the quality control of processed aconite roots and their products.

\section{Background}

Plants of the genus Aconitum are widely distributed across Asia and North America. For over two thousand years, Radix Aconiti Lateralis Preparata (Fuzi, aconite roots) has been used in China to relieve joint pain and treat rheumatic diseases [1]. Studies demonstrated some pharmacological effects of aconite roots, such as analgesia and antiinflammation $[2,3]$. Processing aconite roots is necessary to remove the poisonous diester-diterpene type Aconitum alkaloids including aconitine, mesaconitine and hypaconitine. For example, before used in proprietary herbal products, the toxicity of these alkaloids can be lowered by hydrolysis into much less poisonous benzoylaconines which are the products of deacetylation of the $8 \beta$-acetoxyl [4]. Previously, we established a high-performance liquid chromatography (HPLC) method for determining these 
three toxic Aconitum alkaloids [5]. By comparing their peak areas, we found that benzoylmesaconine (BMA, Figure 1), one of the benzoylaconines, was more abundant than the other two alkaloids in most processed aconite roots [5]. It was reported that BMA at the dosage of $30 \mathrm{mg} /$ $\mathrm{kg}$ (per oral) significantly increased the pain threshold in rats. Its analgesic potency was as potent as that of aconite roots at $1000 \mathrm{mg} / \mathrm{kg}$ (per oral) [6]. Recently, BMA was further shown to have specific cellular immunological activities, antiviral and antifungal activities [7], antinociceptive effects [8], and stimulating activities on cytokines secretion [9]. Due to its abundance and pharmacology, BMA was selected as a single marker compound for the evaluation of the quality of the processed aconite roots and their proprietary herbal products.

The present study developed a suitable analytical method for identification and quantification of BMA to simplify the quality control procedures. Several HPLC methods have been reported [10], none of which is efficient. For instance, the target compounds could not be efficiently separated in acetonitrile. Tetrahydrofuran (THF) was used as the organic component of the mobile phase, but THF would damage the $\mathrm{RP}-\mathrm{C}_{18}$ column when its percentage was over $10 \%$ [10]. In our previous HPLC method, buffer with a $\mathrm{pH}$ value around 10 was used as mobile phase and a special $\mathrm{RP}-\mathrm{C}_{18}$ column was required to stand such an alkaline buffer $(\mathrm{pH}>8)[5,11]$. At the moment, gas chromatography-mass spectrometry (GC-MS) and liquid chromatography-mass spectrometry (LC-MS) methods are applicable, but both require sophisticated sample preparation, such as pre-column derivation and solid phase extraction (SPE) [12-14]. In the present study, we devel-

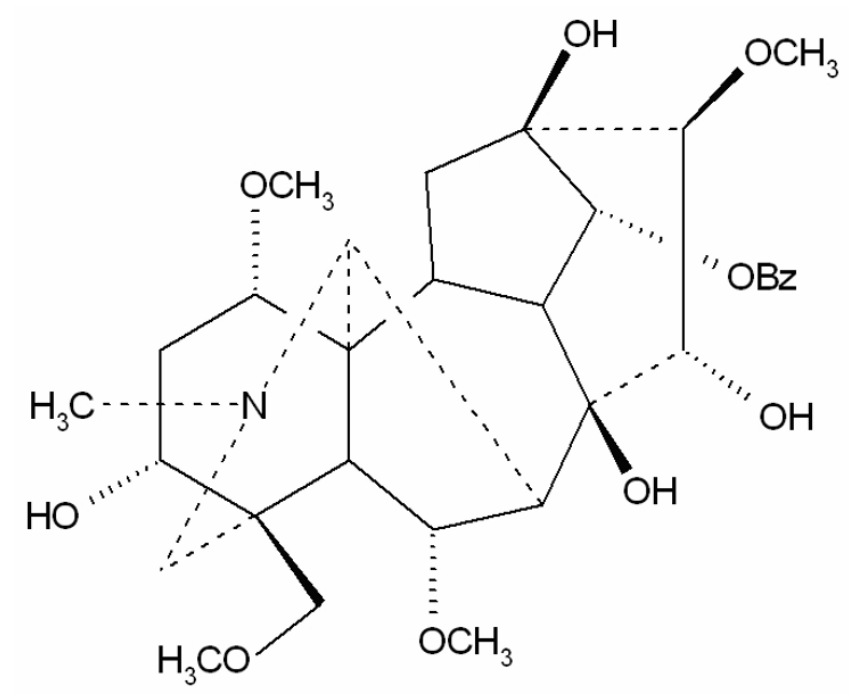

Figure I

Chemical structure of benzoylmesaconine (BMA). oped a simple and reliable HPLC method for quantitative determination of BMA in processed aconite roots and their products.

\section{Methods}

Processed aconite roots and their proprietary products

Nine batches of the processed aconite roots were purchased from herbal medicine markets in Sichuan, Shanxi and Guangdong provinces in China (Table 1).

The Chinese proprietary products containing processed aconite roots used in this study included Guifulizhong Wan (GW, Foshan Fengliaoxiang Pharmaceutical Co, Guangdong, China), Sanqisangyao Capsule (SC, Guangxi Yulin Pharmaceutical Co, Guangxi, China), Haimabushen Wan (HW, Tianjin Zhongxin Pharmaceutical Co, Tianjin, China), and Jinguishengi Wan (JW, Tongrentang Pharmaceutical Co, Beijing, China). The main indications of these products are muscular disorders, joint pain and arthritis. Qingfuguanjieshu Capsule (QC), a new antiarthritic herbal preparation being developed in our research group $[15,16]$, was also used in the present study.

\section{Chemicals and reagents}

Acetonitrile was of HPLC grade (International Laboratory, USA). Twenty-eight per cent (28\%) ammonia solution (AJAX Chemicals, Australia), 95\% ethanol (UNI-CHEM, Hong Kong, China), chloroform (TEDIA, USA), ethyl acetate, triethylamine, $85 \%$ phosphoric acid, diethyl ether anhydrous (International Laboratory, USA) and hydrochloric acid (Merck, Germany) were of guaranteed reagent grade. Deionized water was prepared using a Millipore water purification system (Millipore Corp, USA).

The reference standard for BMA was prepared from mesaconitine (National Institute for the Control of Pharmaceutical and Biological Products, China) which was heated at $100^{\circ} \mathrm{C}$ in dioxane-water $(1: 1)$ for 6 hours and purified by column chromatography and crystallization $[5,11]$. The identity and purity $(\geq 95 \%)$ of BMA were confirmed by TLC (thin-layer chromatography), HPLC, and spectroscopy ( ${ }^{1} \mathrm{H}-\mathrm{NMR}$ and MS) [17].

Table I: BMA content $(\mu \mathrm{g} / \mathrm{g})$ in processed aconite roots $(\mathrm{n}=2)$

\begin{tabular}{lll}
\hline Samples (batch no) & Sample source & Mean $(\mathrm{SD})(\mu \mathrm{g} / \mathrm{g})$ \\
\hline FZ040525-sI & Sichuan province & $13.4(0.5)$ \\
FZ040525-s2 & Sichuan province & $33.5(0.1)$ \\
FZ030II3-0I & Shanxi province & $25.3(0.1)$ \\
FZ030616-0I & Sichuan province & $46.2(1.2)$ \\
FZ030902-0I & Guangdong province & $10.7(0.3)$ \\
FZ040406-0I & Guangdong province & $79.1(1.0)$ \\
FZ040406-03 & Guangdong province & $164.0(3.6)$ \\
FZ040406-07 & Guangdong province & $2.9(0.1)$ \\
FZ040406-II & Guangdong province & $4.6(0.1)$
\end{tabular}




\section{HPLC conditions}

An Agilent 1100 series LC system (Hewlett Packard, USA) was used in the study, which consisted of a G1311A quaternary pump, a G1322A degasser, a G1315A diode-array detector and a G1313A autosampler.

Alltima $\mathrm{RP}_{-} \mathrm{C}_{18}$ (ID $250 \times 4.6 \mathrm{~mm}$, particle size $5 \mu \mathrm{m}$, Alltech Associates, Inc., USA) was used as the stationary phase companied by Alltima RP-C $\mathrm{C}_{18}$ guard column (ID $7.5 \times 4.6 \mathrm{~mm}$ ). Elution of the alkaloids was carried out using a gradient of acetonitrile (A) and buffer solution (B, containing $0.1 \%$ phosphoric acid and $0.1 \%$ triethylamine, adjusted to $\mathrm{pH} 3.0$ with triethylamine) at a flowrate of $1.0 \mathrm{ml} / \mathrm{min}$. The gradient elution of the mobile phase was $13-18 \% \mathrm{~A}$ in $0-20 \mathrm{~min}, 18-21 \% \mathrm{~A}$ in $20-40$ $\min , 21-22 \% \mathrm{~A}$ in $40-45 \mathrm{~min}$ and $22-70 \% \mathrm{~A}$ in $45-50$ min. Detection was carried out at $240 \mathrm{~nm}$ with a reference wavelength of $550 \mathrm{~nm}$ at room temperature. The injection volume was $20 \mu \mathrm{l}$ for all HPLC runs.

\section{Preparation of sample solutions}

Processed aconite roots were pulverized into powder, passed through a $0.45 \mathrm{~mm}$ sieve, and stored in a desiccator. The powder (1.5 g) was extracted with $50 \%$ ethanol three times $(4.0 \mathrm{ml}, 4.0 \mathrm{ml}$ and $2.0 \mathrm{ml}$ respectively and 60 min each time) by sonication (Branson 5210 Ultrasonicator, Balkowitsch Enterprises, Inc., USA) at room temperature, and then vortexed for $2 \mathrm{~min}$ respectively. The mixture was centrifuged for $10 \mathrm{~min}$ at $3000 \mathrm{rpm}$ (Eppendorf 5810 Centrifuge, Scios, Inc., USA). The supernatants were combined and transferred to a $10.0 \mathrm{ml}$ volumetric flask, with 50\% ethanol making up the volume.

Powdered pills or contents of capsules of the proprietary products (approximately $1.0 \mathrm{~g}$ ) were accurately weighed. Each sample was dissolved in $10 \mathrm{ml}$ of $\mathrm{HCl}$ solution $(0.05$ M) by sonication for $60 \mathrm{~min}$, and extracted with ethyl acetate three times $(10 \mathrm{ml}$ each time). The acidic aqueous solution was separated and basified with $600 \mu \mathrm{l}$ of $28 \%$ ammonia solution and further extracted with $10 \mathrm{ml}$ of mixed solvents of diethyl ether and ethyl acetate (1:1) three times by vortexing for $2 \mathrm{~min}$. The resulting mixtures were centrifuged at $3000 \mathrm{rpm}$ (Eppendorf 5810 Centrifuge, Scios Inc, USA) for $5 \mathrm{~min}$ and the combined supernatants were evaporated to dryness under air stream. The residue was dissolved in $1.0 \mathrm{ml}$ of $\mathrm{HCl}$ solution $(0.01 \mathrm{M})$ by sonication for $1 \mathrm{~min}$.

\section{Preparation of standard solutions}

BMA was accurately weighed and dissolved in $\mathrm{HCl}$ solution $(0.01 \mathrm{M})$ to produce a stock standard solution at a final concentration of $0.4084 \mathrm{mg} / \mathrm{ml}$. This stock solution was used to prepare standard solutions for method validation and calibration curves. The standard solutions were stored at $4^{\circ} \mathrm{C}$ and remained stable for at least one month.
Calibration curves were established at seven concentration $(\mu \mathrm{g} / \mathrm{ml})$ points of $4.08,10.21,20.42,40.84,81.68$, 122.52 and 204.20 respectively. For recovery test, a standard solution was prepared at a concentration of $12.25 \mu \mathrm{g} /$ $\mathrm{ml}$.

\section{Validation of the HPLC method \\ Precision}

Expressed as relative standard deviations (RSDs), precision was evaluated by HPLC runs with standard solutions at three concentrations under the optimal condition five times in one day for intra-day variation test and twice a day on three consecutive days for inter-day variation test.

\section{Repeatability}

Six aliquots of QC were conducted for repeatability test. The processing of the aliquots followed the same method described in the section of preparation of sample solutions. The RSDs of the six aliquots were calculated for evaluation of repeatability.

\section{Recovery}

$\mathrm{HCl}$ solution $(10 \mathrm{ml}, 0.05 \mathrm{M})$ was added to accurately weighted QC (approximately $0.1 \mathrm{~g}$ ) in which the content of BMA was known. The sample solution was spiked with $1000 \mu \mathrm{l}$ of the standard solution of BMA for recovery test. The processing of the prepared samples $(n=6)$ followed the method described in the section of preparation of sample solutions.

\section{Statistical analysis}

Data analysis, including calculation of standard deviation (SD) and linear regression, was conducted using Excel 2003 (Microsoft, USA). P values less than 0.05 were considered as statistically significant.

\section{Results and discussion Optimization of HPLC conditions}

The use of phosphoric acid $(0.1 \%)$ with triethylamine as the mobile phase produced better symmetry of the peak in the chromatogram. A gradient elution program was developed to isolate BMA and to determine the content of BMA in the proprietary products. Figure 2 shows a chromatogram of BMA $(35 \mu \mathrm{g} / \mathrm{ml})$ in standard solution. Figure 3 shows a chromatogram (detection at $240 \mathrm{~nm}$ ) of BMA in $50 \%$ ethanol extract of processed aconite roots. The results indicate that the retention time (43.5 $\mathrm{min}$ ) of BMA in processed aconite roots (Figure 3 ) and in QC preparation (Figure 4) was consistent with that of BMA in standard solution (Figure 2).

By adjusting the triethylamine content to obtain the $\mathrm{pH}$ values from 2.3 to 5.6, we investigated the $\mathrm{pH}$ dependence of the retention time. The results show BMA retention time remained relatively stable in the range of 42-44 min 


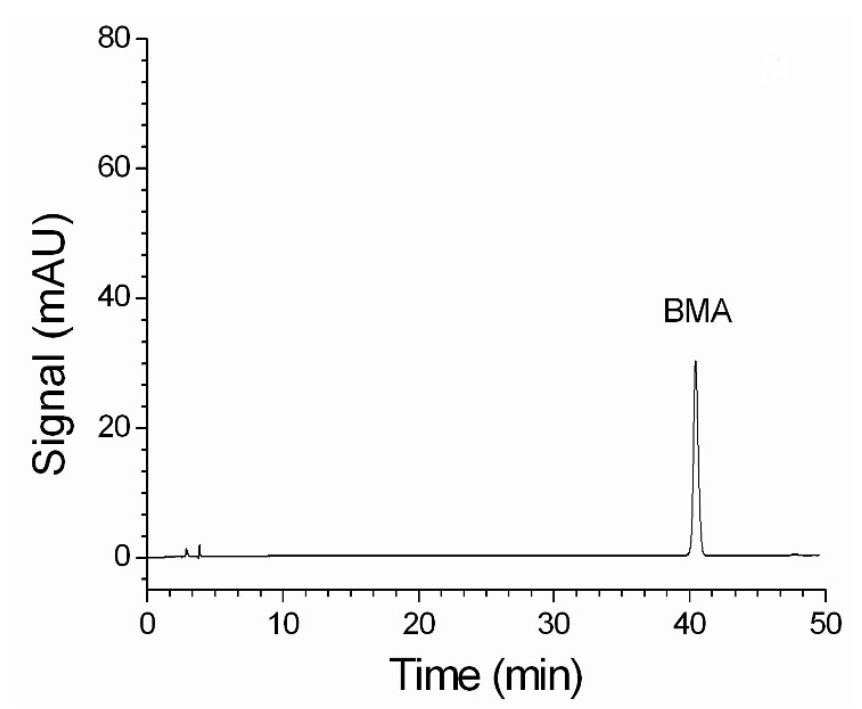

Figure 2

An HPLC chromatogram of BMA standard solution.

as $\mathrm{pH}$ value increased (Figure 5). Due to a long elution time and drifting baseline at $\mathrm{pH}$ values below 2.6 and above 4.9 respectively, the HPLC runs in the present study were carried out at pH 3.0 for best effect.

\section{Optimization of extraction conditions}

Common extracting solvents including water and aqueous ethanol (95\%, $75 \%, 50 \%$ and $25 \%$ ) were evaluated for their efficiency in extracting BMA from processed aconite roots. Since BMA is an alkaloid, an acidic extraction solvent $(0.01 \mathrm{M} \mathrm{HCl})$ was also tested. The results show

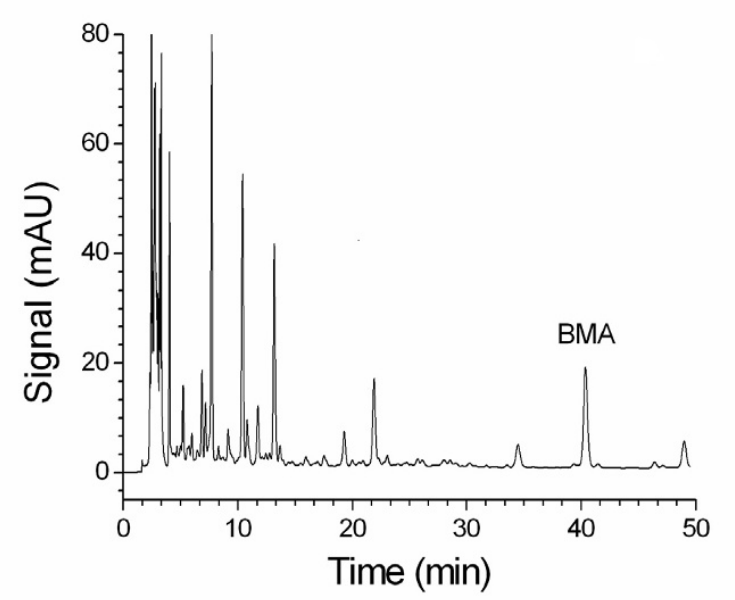

Figure 3

An HPLC chromatogram of BMA in processed aconite roots (batch no 040406-0I).

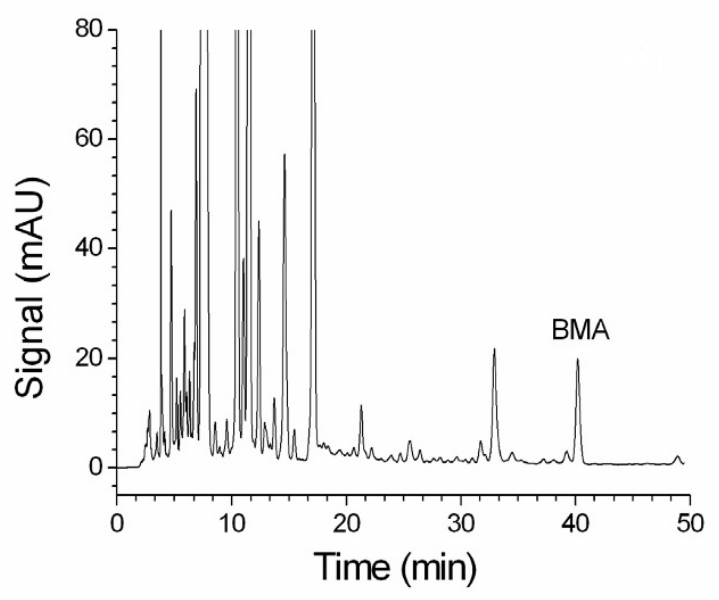

Figure 4

An HPLC chromatogram of BMA in QC.

that the extraction efficiency of these solvents was in the following order: $50 \%$ ethanol $>25 \%$ ethanol $>0.01 \mathrm{M}$ $\mathrm{HCl}>75 \%$ ethanol > $95 \%$ ethanol. For highest efficiency, $50 \%$ ethanol was selected for extracting BMA in processed aconite roots in the present study (Table 2).

As a single-step extraction with $50 \%$ ethanol contained many impurities, we developed a multi-step extraction to remove some non-alkaloid components in the extracts. Powdered proprietary products were dissolved in $10 \mathrm{ml}$ of $\mathrm{HCl}$ solution $(0.05 \mathrm{M})$ by sonication for $60 \mathrm{~min}$, which were subsequently extracted with ethyl acetate for three

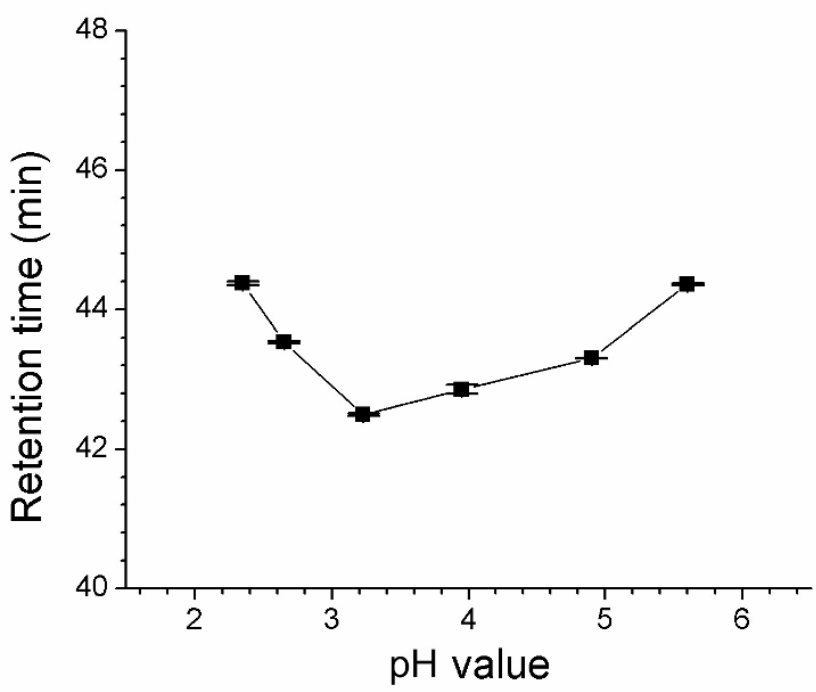

Figure 5

Relation of $\mathrm{pH}$ and the retention time of BMA. 
Table 2: Efficiency of different solvents in extracting the content of BMA $(\mu \mathrm{g} / \mathrm{g})$ from processed aconite roots $(n=2)$

\begin{tabular}{ccc}
\hline Solvents & Mean $(\mathrm{SD})(\mu \mathrm{g} / \mathrm{g})$ & RSD (\%) \\
\hline $0.01 \mathrm{M} \mathrm{HCl}$ & $20.8(0.3)$ & $1.24 \%$ \\
$25 \%$ ethanol & $22.7(0.0)$ & $0.10 \%$ \\
$50 \%$ ethanol & $25.3(0.1)$ & $0.45 \%$ \\
$75 \%$ ethanol & $14.8(0.3)$ & $0.18 \%$ \\
$95 \%$ ethanol & $0.55(0.06)$ & $10.98 \%$ \\
\hline
\end{tabular}

Note: Sample FZ030 I I3-0I was used in this experiment.

times (10 ml each time). The acidic aqueous solutions were then basified with $600 \mu$ l of $28 \%$ ammonia solution and further extracted with $10 \mathrm{ml}$ of mixed solvents of diethyl ether and ethyl acetate (1:1) for three times. The recovery rate of BMA was $90.64 \%$ (SD 0.58\%) when diethyl ether was the extraction solvent. The recovery rate was nearly perfect when ethyl acetate was the extraction solvent; however, there was a small interference peak at the retention time of $43 \mathrm{~min}$. Thus, a mixed solvent of diethyl ether and ethyl acetate (1:1) was used, whereby the recovery rate was $96.95 \%$ (SD 1.01\%) and no interference peak was observed.

\section{Validation of the HPLC method}

The BMA peaks in processed aconite roots and in their proprietary products were identified through comparison of the retention times and UV spectra with those of the reference standards. Peak purity was confirmed by data from a photodiode array detector (DAD). Furthermore, a comparative study was carried out with QC samples and QC without processed aconite roots (negative control). There

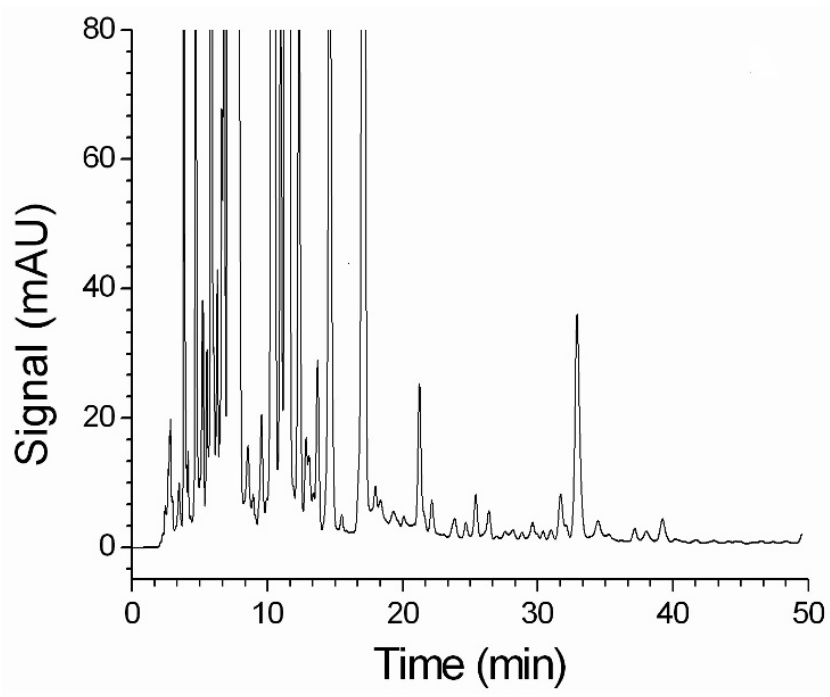

Figure 6

An HPLC chromatogram of QC product without processed aconite roots (negative control). is no peak corresponding to BMA in the chromatogram of the negative control (Figure 6).

The linearity of the BMA concentrations $(\mu \mathrm{g} / \mathrm{ml})$ versus peak areas was investigated in the range of 4.08-204.20 $\mu \mathrm{g} / \mathrm{ml}$; the result is expressed as the value of the coefficient of determination $\left(\mathrm{R}^{2}\right)$. The following equation was obtained: $\mathrm{Y}=18.518 \mathrm{X}+5.651\left(\mathrm{R}^{2}=0.9998, \mathrm{P}<0.001\right)$.

The limit of detection, defined as the amount of the compounds needed to produce a signal (S) at least three times larger than noise $(\mathrm{N})$, was determined to be $8 \mathrm{ng}$ for BMA with an injection volume of $20 \mu \mathrm{l}$.

The precision of the intra-day (five times per day) and inter-day (twice a day for three consecutive days) data was indicated by RSDs which were less than $1.36 \%$ for BMA at three concentrations (Table 3 ). These results suggest that $\mathrm{BMA}$ is stable in acidic solution $(0.01 \mathrm{M} \mathrm{HCl})$. The accuracy was determined by back calculation of precision samples at three concentrations against a calibration curve prepared each day.

The RSDs of the repeatability test were less than $0.29 \%$ for BMA at $132.8 \mu \mathrm{g} / \mathrm{g}$ (SD $0.4 \mu \mathrm{g} / \mathrm{g}$ ) in the QC samples ( $\mathrm{n}=$ 6 ). The average recovery rate obtained was $96.95 \%$ (SD $1.01 \%)$ with RSDs of $0.01 \%(n=6)$. This chromatographic system is suitable for quantitative determination of BMA in processed aconite roots and their proprietary products.

\section{Quantitative determination of BMA}

The content of BMA in the five proprietary products, and nine batches of processed aconite roots were determined using the established HPLC method described above. The content of BMA (Tables 1 and 4) was calculated with the regression equations obtained from the calibration curves.

Significant variations in terms of BMA content were found among different batches of processed aconite roots and among different proprietary products (Tables 1 and 4 ). Differences in species, places of origin, processing methods of the raw materials and pharmaceutical manufacturing processes may all contribute to the variations.

As herbal products containing processed aconite roots are increasingly used for anti-inflammation and analgesia, simple and reliable methods for quality control of these products are urgently needed. It was reported that BMA was effective in anti-inflammation and analgesia in animals $[2,6]$. Thus, BMA was selected as a marker compound to assess the quality of processed aconite roots and their proprietary products. 
Table 3: Precision of the intra-day and inter-day measurements

\begin{tabular}{cccccc}
\hline Conc. $\mu \mathrm{g} / \mathrm{ml}$ & \multicolumn{2}{c}{ Intra-day a } & \multicolumn{2}{c}{ Inter-day ${ }^{\mathrm{b}}$} \\
& Peak areas Mean (SD) & RSD \% & Precision \% & Peak areas Mean (SD) & RSD \% Precision \% \\
\hline \multirow{2}{*}{ Low (4.08) } & $63.0(0.8)$ & 1.36 & $95.90 \%$ & $65.3(0.5)$ & 0.88 \\
Mid (20.42) & $303.1(0.4)$ & 0.15 & $95.48 \%$ & $309.9(1.8)$ & $95.08 \%$ \\
High (122.5) & $1870.0(7.5)$ & 0.39 & $97.55 \%$ & $1890.0(17.8)$ & 0.60 \\
\hline
\end{tabular}

Note: ${ }^{a}: \mathrm{n}=5$; b: $\mathrm{n}=6$ (twice a day for three consecutive days).

In the present study, extraction method was optimized. Results from the recovery test show that the use of two solvents is better than that of a single solvent in preparing samples of proprietary products.

In the analysis of Aconitum alkaloids using a reversed phase $\mathrm{C}_{18}$ HPLC column, tailing peaks are usually caused by the retention effects of free silanol groupings. Instead of using THF as an organic solvent or basic buffer in the mobile phase, we used triethylamine to improve separation and peak shapes. Moreover, this HPLC method should be easily repeated in other labs. The present study also indicates that the retention volumes of BMA remained relatively unchanged over an acidic $\mathrm{pH}$ region (Figure 5).

\section{Conclusion}

Method validation data indicate that the developed HPLC method as described in this paper is reliable, reproducible and accurate for the determination of BMA in processed aconite roots and their products. The results also show that the content of BMA varied significantly in different batches of the processed aconite roots including five proprietary products. This method is suitable for routine assessment of the quality of processed aconite roots and their products.

\section{Abbreviations}

BMA: benzoylmesaconine; DAD: photodiode array detector; GC-MS: gas chromatography-mass spectrometry; HPLC: high-performance liquid chromatography; LC-MS: liquid chromatography-mass spectrometry; RSDs: relative

Table 4: BMA content $(\mu \mathrm{g} / \mathrm{g})$ in five herbal proprietary products containing processed aconite roots $(n=2)$

\begin{tabular}{cccc}
\hline Product & Batch no & Dosage (g/day) & Mean (SD) $(\mu g / g)$ \\
\hline GW & 0405005 & 18 & $6.9(0.1)$ \\
SC & 20030801 & 2.25 & $245.0(0.7)$ \\
HW & 030769 & 5.4 & $58.0(0.2)$ \\
JW & 3030904 & 10 & $6.0(0.0)$ \\
QC & 20040901 & 8.5 & $132.8(0.4)$ \\
\hline
\end{tabular}

Note: GW: Guifulizhong Wan, SC: Sanqisangyao Capsule, HW: Haimabushen Wan, JW: Jinguishenqi Wan, QC: Qingfuguanjieshu Capsule standard deviations; SD: standard deviation; SPE: solid phase extraction; THF: tetrahydrofuran

\section{Competing interests}

The authors declare that they have no competing interests.

\section{Authors' contributions}

YX contributed to the conception and design of the study, carried out the experimental work, and drafted the manuscript, ZJ contributed to the design of the study and helped revise the manuscript, $\mathrm{HZ}$ participated in the design of the study and performed statistical analysis, YW and ZL participated in the experimental work of Qingfuguanjieshu Capsule and helped with data acquisition, HX contributed to the design of the study, LL, the principal investigator of the project, contributed to the conception and design of the study, revised and finalized the manuscript. All authors read and approved the final version of the manuscript.

\section{Acknowledgements}

This work was funded by the Hong Kong Jockey Club Charities Trust.

\section{References}

I. Bisset NG: Arrow poisons in China (Part II): Aconitum - botany, chemistry, and pharmacology. I Ethnopharmacol 198I, 4:247-336.

2. Hikino H, Konno C, Takata H, Yamada Y, Yamada C, Ohizumi $Y$, Sugio K, Fujimura $\mathrm{H}$ : Anti-inflammatory principles of Aconitum roots. J Pharmacobiodyn 1980, 3(10):5। 4-525.

3. Gutser UT, Friese J, Heubach JF, Matthiesen T, Selve N, Gleitz J: Mode of antinociceptive and toxic action of alkaloids of Aconitum sepc. Naunyn Schmiedebergs Arch Pharmacol 1998, 357:39-48.

4. Xu Q, Zhang F, Xiao HB, Liang XM, Kettrup A: Study on detoxification of main alkaloids in Aconitum by high performance liquid chromatography -atmospheric pressure chemical ionization mass spectrometry. Fresenius Environ Bull 2005, 14:194-198

5. Jiang ZH, Xie Y, Zhou H, Liu ZQ, Wong YF, Cai X, Xu HX, Liu L: Quantication of Aconitum alkaloids in aconite roots by a modified RP-HPLC method. Phytochem Anal 2005, 16:415-421.

6. Suzuki $Y$, Hayakawa $Y$, Oyama $T$, Isono $T$, Ohmiya $Y$, Ikeda $Y$, Asami A, Noguchi M: Analgesic effect of benzoylmesaconine. Nippon Yakurigaku Zasshi 1993, 102:399-404.

7. Kobayashi M, Mori K, Kobayashi H, Pollard RB, Suzuki F: The regulation of burn-associated infections with herpes simplex virus type I or Candida albicans by a non-toxic aconitinehydrolyzed, benzoylmesaconine. Part I: antiviral and antifungal activities in thermally injured mice. Immun Cell Bio 1998 , 76(3):202-208.

8. Suzuki $Y$, Oyama T, Ishige A, Isono $T$, Asami A, Ikeda $Y$, Noguchi M, Ohmiya $Y$ : Antinociceptive mechanism of the aconitine alkaloids mesaconitine and benzoylmesaconine. Planta Med 1994, 60:391-394. 
9. Kobayashi M, Takahashi H, Herndon DN, Pollard RB, Suzuki F: Soluble IL-4 receptor improves the skin-graft-associated cytomegalovirus infection in thermally injured mice. Burns 2003, 29:37-42.

10. Hikino H, Konno C: Determination of aconitine alkaloids by high-performance liquid chromatography. J Chromatogr 198I, 2II:I23-128.

II. Xie Y, Jiang ZH, Zhou H, Xu HX, Liu L: Simultaneous determination of six Aconitum alkaloids in proprietary Chinese medicines by high-performance liquid chromatography. J Chromatogr A 2005, 1093: 195-203.

12. Ohta H, Seto Y, Tsunoda N, Takahashi Y, Matsuura K, Ogasawara K: Determiantion of Aconitum alkaloids in blood and urine samples. II. Capillary liquid chromatographic-frit fast atom bombardment mass spectrometric analysis. J Chromatogr B 1998, 7| 4:2I5-22I.

13. Ohta H, Seto Y, Tsunoda N: Determination of Aconitum alkaloids in blood and urine samples. I. High performance liquid chromatographic separation, solid-phase extraction and mass spectrometric confirmation. J Chromatogr B 1997, 69I:35I-356.

14. Ito K, Satoshi T, Funayama M, Mizugaki M: Quantitative analysis of Aconitum alkaloids in the urine and serum of a male attempting suicide by oral intake of aconite extract. J Anal Toxicol 2000, 24:348-340.

15. Cai X, Zhou H, Wong YF, Xie Y, Liu ZQ, Jiang ZH, Bian ZX, Xu HX, Liu L: Suppressive effects of QFGJS, a preparation from an anti-arthritic herbal formula, on rat experimental adjuvantinduced arthritis. Biochem Biophys Res Commun 2005, 337:586-594.

16. Zhou H, Wong YF, Cai X, Liu ZQ, Jiang ZH, Bian ZX, Xu HX, Liu L: Suppressive Effects of JCICM-6, the Extract of an Antiarthritic Herbal Formula, on the Experimental Inflammatory and Nociceptive Models in Rodents. Biol Pharm Bull 2006, 29:253-260.

17. Pelletier SW: Alkaloids, Chemical and Biological Perspectives Volume 7. New York: Springer-Verlag; 1991:390-397.

Publish with Bio Med Central and every scientist can read your work free of charge

"BioMed Central will be the most significant development for disseminating the results of biomedical research in our lifetime. "

Sir Paul Nurse, Cancer Research UK

Your research papers will be:

- available free of charge to the entire biomedical community

- peer reviewed and published immediately upon acceptance

- cited in PubMed and archived on PubMed Central

- yours - you keep the copyright 